\title{
THE EQUIVALENCE OF VARIOUS DEFINITIONS FOR A PROPERLY INFINITE VON NEUMANN ALGEBRA TO BE APPROXIMATELY FINITE DIMENSIONAL
}

\author{
G. A. ELLIOTT AND E. J. WOODS
}

\begin{abstract}
If a properly infinite von Neumann algebra on a separable Hilbert space is approximately finite dimensional with respect to the *ultrastrong topology, that is, if any finite number of elements may be approximated *-ultrastrongly by elements of a finite-dimensional sub *algebra, then the algebra may be expressed as the bicommutant of an increasing sequence of factors of type $I_{2^{n}}$.
\end{abstract}

In [2] Murray and von Neumann introduced and proved the equivalence of several definitions that a factor of type $\mathrm{II}_{1}$ on a separable Hilbert space be approximately finite dimensional. (They used the slightly elliptic term "approximately finite". When a factor of type $\mathrm{II}_{1}$ came itself to be called "finite", the term "hyperfinite" was introduced as a replacement [1]. This term has since been used to describe certain infinite factors, a situation in which it is not quite appropriate. We use instead the term "approximately finite dimensional".)

In this paper we shall prove that the obvious translations of the definitions of Murray and von Neumann to the infinite case remain equivalent, and that it is not necessary in this case to restrict attention to factors.

LEMMA 1. Let $A$ be a properly infinite von Neumann algebra, $\xi$ a vector, $\varepsilon>0$. Then there exists $u \in A$ such that

(i) $u^{*} u=1$ and $1-u u^{*}$ is equivalent to 1 ;

(ii) $\|(1-u) \xi\| \leqslant \varepsilon,\left\|(1-u)^{*} \xi\right\| \leqslant \varepsilon,\left\|\left(1-u u^{*}\right) \xi\right\| \leqslant \varepsilon$.

Proof. Choose a projection $f$ equivalent to 1 and such that $\|f \xi\| \leqslant \varepsilon / 2$. (If $1=\sum f_{n}$ with each $f_{n}$ equivalent to 1 , choose $f$ to be $\sum_{n=n_{0}}^{\infty} f_{n}$ with $n_{0}$ large.) Choose a projection $e \leqslant f$ such that $e$ and $f-e$ are equivalent. (If $f$ $=\sum_{n=n_{0}}^{\infty} f_{n}$ with each $f_{n}$ equivalent to 1 , choose $e$ to be $\sum_{n=n_{0}}^{\infty} f_{2 n}$.) Then $e$ and $f-e$ are both equivalent to $f$; in particular, there exists $v$ with $v^{*} v=f$ and $v v^{*}=f-e$. Set $1-f+v=u$. Then $(1-u)=f(1-u) f$, whence

$$
\|(1-u) \xi\| \leqslant \varepsilon, \quad\left\|(1-u)^{*} \xi\right\| \leqslant \varepsilon .
$$

Moreover, $u^{*} u=1$ and $1-u u^{*}=e$. Since $\|e \xi\| \leqslant\|f \xi\| \leqslant \varepsilon / 2$, we have (ii). Since $e$ is equivalent to $f$, and hence to 1 , we have (i).

Received by the editors January 12, 1976.

AMS (MOS) subject classifications (1970). Primary 46L10.

Copyright $\odot$ 1977, American Mathematical Society 
Lemma 2. Let $A, B$ be von Neumann algebras, $B \subset A$, $\xi$ a separating vector for $A$, and $x \in A$. If there exists a sequence $\left(x_{n}\right)$ in $B$ such that $\left\|\left(x_{n}-x\right) \xi\right\| \rightarrow 0$ and $\left\|\left(x_{n}^{*}-x^{*}\right) \xi\right\| \rightarrow 0$ then $x \in B$.

Proof. It follows that $\left\|\left[\left(x_{n} \pm x_{n}^{*}\right)-\left(x \pm x^{*}\right)\right] \xi\right\| \rightarrow 0$, and hence it is sufficient to consider the case where the $x_{n}, x$ are selfadjoint. Since $\left(x_{n}\right)$ may be unbounded, it does not follow that $x_{p} \rightarrow x$ strongly. To avoid this difficulty we use the resolvents. Since $\left\|\left(x_{n}+i\right)^{-1}\right\| \leqslant 1$, it follows from the identity

$$
\left(x_{n}+i\right)^{-1}-(x+i)^{-1}=\left(x_{n}+i\right)^{-1}\left(x-x_{n}\right)(x+i)^{-1}
$$

that $\left\|\left[\left(x_{n}+i\right)^{-1}-(x+i)^{-1}\right] \eta\right\| \rightarrow 0$ where $\eta=(x+i) \xi$. Since $\xi$ is cyclic for $A^{\prime}$ and $x+i$ is invertible in $A$, it follows that $\eta$ is also cyclic for $A^{\prime}$. It now follows easily that $\left(x_{n}+i\right)^{-1} \rightarrow(x+i)^{-1}$ strongly.

TheOREM 3. Let $A$ be a properly infinite von Neumann algebra acting on a separable Hilbert space. Then the following three conditions are equivalent.

(i) For any $x_{1}, \ldots, x_{n} \in A$ and any *-ultrastrong neighbourhood $V$ of 0 in $A$ there exist a finite-dimensional sub *-algebra $N$ of $A$ and $y_{1}, \ldots, y_{n} \in N$ such that $x_{i}-y_{i} \in V, i=1, \ldots, n$.

(ii) Condition (i) holds and $N$ may be chosen to be a factor of type $\mathrm{I}_{2^{k}}$.

(iii) There exists an increasing sequence of factors $N_{k}$ of type $\mathrm{I}_{2^{k}}$ such that $A=\left(\cup N_{k}\right)^{\prime \prime}$.

Proof. (i) $\Rightarrow$ (ii). Let $x_{1}, \ldots, x_{n} \in A$. Let $V$ be a *-ultrastrong neighbourhood of 0 in $A$. We may suppose, replacing the Hilbert space on which $A$ acts by its tensor product with another Hilbert space, and $A$ by $A \otimes 1$, that $V$ is determined by a single vector $\xi$, that is, that

$$
V=\left\{a \in A ;\|a \xi\| \leqslant 1,\left\|a^{*} \xi\right\| \leqslant 1\right\}
$$

Replacing $V$ by a smaller neighbourhood, we may suppose that $\xi$ is separating for $A$.

By (i), there exist a finite-dimensional sub *-algebra $M$ of $A$, and $z_{1}, \ldots, z_{n}$ $\in M$ such that $x_{i}-z_{i} \in 3^{-1} V, i=1, \ldots, n$.

Since $\xi$ is separating, there exists $\delta>0$ such that if $u^{*} u=1, u-1 \in \delta V$, $u^{*}-1 \in \delta V$ then $u z_{i} u^{*}-z_{i} \in 3^{-1} V, i=1, \ldots, n$. Choose $u$ as given by Lemma 2 with $\varepsilon=\min \left(\delta,\left(3 \sup \left\|z_{i}\right\|\right)^{-1}\right)$; then $u^{*} u=1,1-u u^{*}$ is equivalent to 1 , and

$$
\begin{gathered}
u z_{i} u^{*}-z_{i} \in 3^{-1} V, \quad i=1, \ldots, n, \\
1-u u^{*} \in\left(3 \sup \left\|z_{i}\right\|\right)^{-1} V .
\end{gathered}
$$

Write $M$ as a direct sum of factors $N_{r}$, of type $I_{n_{r}}$, with unit $e^{(r)}, r=1, \ldots$, $R$. Write

$$
z_{i}=\sum_{r, p, q} z_{i, p q}^{(r)} e_{p q}^{(r)}
$$

where $\left(e_{p q}^{(r)}\right)$ is a complete system of matrix units for $N_{r}$, and $z_{i, p q}^{(r)} \in \mathbf{C}$. Choose $k=1,2, \ldots$ such that $2^{k} \geqslant t=\sum_{r=1}^{R} n_{r}$. Partition $1-u u^{*}$ into $2^{k}$ mutual- 
ly equivalent projections. Denote the first $t$ of these by $\varepsilon_{p p}^{(r)}, p=1, \ldots, n_{r}, r$ $=1, \ldots, R$.

For each $r=1, \ldots, R$, extend $\left(\varepsilon_{p p}^{(r)}\right)_{p=1, \ldots, n_{r}}$ to a system of matrix units $\left(\varepsilon_{p q}^{(r)}\right)_{p, q=1, \ldots, n_{r}}$. Set

$$
\left(u e_{p q}^{(r)} u^{*}+\varepsilon_{p q}^{(r)}\right)=\left(f_{p q}^{(r)}\right)
$$

then $\left(f_{p q}^{(r)}\right)$ is a system of matrix units. Set

$$
\sum_{r, p, q} z_{i, p q}^{(r)} f_{p q}^{(r)}=y_{i}, \quad i=1, \ldots, n
$$

Then for each $i=1, \ldots, n$,

$$
\begin{gathered}
\left\|\left(u z_{i} u^{*}-y_{i}\right) \xi\right\|=\left\|\sum_{r, p, q} z_{i, p q}^{(r)} \varepsilon_{p q}^{(r)} \xi\right\| \leqslant\left\|z_{i}\right\|\left\|\sum_{r, p} \varepsilon_{p p}^{(r)} \xi\right\| \\
\leqslant\left\|z_{i}\right\|\left\|\left(1-u u^{*}\right) \xi\right\| \leqslant 3^{-1} ; \\
\left\|\left(u z_{i}^{*} u^{*}-y_{i}\right) \xi\right\| \leqslant\left\|z_{i}^{*}\right\|\left\|\left(1-u u^{*}\right) \xi\right\| \leqslant 3^{-1} ; u z_{i} u^{*}-y_{i} \in 3^{-1} V .
\end{gathered}
$$

Hence,

$$
\begin{aligned}
x_{i}-y_{i}= & \left(x_{i}-z_{i}\right)+\left(z_{i}-u z_{i} u^{*}\right)+\left(u z_{i} u^{*}-y_{i}\right) \\
& \in 3^{-1} V+3^{-1} V+3^{-1} V=V, \quad i=1, \ldots, n .
\end{aligned}
$$

Since the projections obtained from the partition of $1-u u^{*}$ are all equivalent to 1 , the projections $f_{p p}^{(r)}$ and the remaining $2^{k}-t$ projections are mutually equivalent. Hence the systems of matrix units $\left(f_{p q}^{(r)}\right), r=1, \ldots, R$, can be extended to a common system of matrix units, the sum of whose projections is 1 . The linear span of these matrix units is a factor of type $I_{2^{k}}$, which is contained in $A$ and contains the above $y_{i}$, and therefore satisfies the requirements for $N$.

(ii) $\Rightarrow$ (iii). The argument is basically the same as in the case that $A$ is of type $\mathrm{II}_{1}$. It is enough to construct an increasing sequence of factors $N_{k_{n}}$ of type $\mathrm{I}_{2^{k_{n}}}$ such that $A=\left(\cup N_{k_{n}}\right)^{\prime \prime}$, and we shall do this inductively.

Choose a sequence $\left(x_{1}, x_{2}, \ldots\right) *$-ultrastrongly dense in $A$. We may suppose that there exists a vector $\xi$ separating for $A$. Fix $n=1,2, \ldots$, and assume that there is a factor $N_{k_{n}} \subset A$ of type $\mathrm{I}_{2^{k_{n}}}$ such that for suitable $y_{1}^{(n)}, \ldots, y_{n}^{(n)}$ in $N_{k_{n}}$,

$$
\left\|\left(x_{i}-y_{i}^{(n)}\right) \xi\right\| \leqslant n^{-1}, \quad\left\|\left(x_{i}-y_{i}^{(n)}\right)^{*} \xi\right\| \leqslant n^{-1}, \quad i=1, \ldots, n .
$$

Choose a complete system of matrix units $\left(e_{p q}\right)$ for $N_{k_{n}}$. Then $e_{11} A e_{11}$ satisfies (ii) in place of $A$, as $e_{11}$ is equivalent to 1 and so $e_{11} A e_{11}$ is isomorphic to $A$. In particular, there exists a factor $N \subset e_{11} A e_{11}$ of type $\mathrm{I}_{2^{k}}$ (with unit $e_{11}$ ) such that for suitable $y_{1, p q}, \ldots, y_{n+1, p q} \in N$, 


$$
\begin{aligned}
\left\|\left(e_{1 p} x_{i} e_{q 1}-y_{i, p q}\right) e_{1 q} \xi\right\| & \leqslant(n+1)^{-1} k_{n}^{-2}, \\
\left\|\left(e_{1 p} x_{i} e_{q 1}-y_{i, p q}\right)^{*} e_{1 p} \xi\right\| & \leqslant(n+1)^{-1} k_{n}^{-2}, \\
i & =1, \ldots, n+1, \quad p, q=1, \ldots, k_{n} .
\end{aligned}
$$

Set

$$
\sum_{p, q} e_{p 1} y_{i, p q} e_{1 q}=y_{i}^{(n+1)}, \quad i=1, \ldots, n+1 .
$$

Then for each $i=1, \ldots, n+1, y_{i}^{(n+1)}$ is in the algebra $N_{k_{n+1}}$ generated by $N_{k_{n}}$ and $N$, a factor of type $\mathrm{I}_{2^{k_{n}+k}}$, and for each $i=1, \ldots, n$,

$$
\begin{aligned}
\left\|\left(x_{i}-y_{i}^{(n+1)}\right) \xi\right\| & =\left\|\sum_{p, q} e_{p 1}\left(e_{1 p} x_{i} e_{q 1}-y_{i, p q}\right) e_{1 q} \xi\right\| \\
& \leqslant \sum_{p, q}(n+1)^{-1} k_{n}^{-2}=(n+1)^{-1} \\
\left\|\left(x_{i}-y_{i}^{(n+1)}\right)^{*} \xi\right\| & =\left\|\sum_{p, q} e_{q 1}\left(e_{1 p} x_{i} e_{q 1}-y_{i, p q}\right)^{*} e_{1 p} \xi\right\| \\
& \leqslant \sum_{p, q}(n+1)^{-1} k_{n}^{-2}=(n+1)^{-1} .
\end{aligned}
$$

This shows that there exist an increasing sequence of factors $N_{k_{n}} \subset A$ of type $\mathrm{I}_{2^{k_{n}}}$ and a separating vector $\xi$ for $A$ such that the hypotheses of Lemma 2 are satisfied for all $x \in A$ with $B=\left(\cup N_{k_{n}}\right)^{\prime \prime}$. Hence $A=\left(\cup N_{k_{n}}\right)^{\prime \prime}$.

(iii) $\Rightarrow$ (i). This follows from the bicommutant theorem.

\section{REFERENCES}

1. J. Dixmier, Les algèbres d'opérateurs dans l'espace hilbertien (algèbres de von Neumann), 2ième éd., Gauthier-Villars. Paris, 1969. MR 50 \#5482.

2. F. J. Murray and J. von Neumann, On rings of operators. IV, Ann. of Math. (2) 44 (1943), 716-808. MR 5, 101.

Mathematics Institute, University of Copenhagen, Copenhagen, Denmark

Department of Mathematics, Queen's University, Kingston, Ontario, Canada 